Gynäkol Geburtshilfliche Rundsch 1996;36:229-230

\title{
Mitteilungen aus Osterreich
}

Österreichische Gesellschaft für Gynäkologie und Geburtshilfe (ÖGGG)

Weitere Veranstaltungen in Osterreich

In der ordentlichen Vollversammlung der Österreichischen Gesellschaft für Gynäkologie und Geburtshilfe (ÖGGG) wurde Herr Prim. Univ. Prof. Dr. Heinz Salzer am 10. Dezember 1996 in Wien zum Präsident elect der Gesellschaft gewählt. Seine Amtszeit als President der ÖGGG ist demnach der Zeitraum 1998/99.

Tagung

29.5.-31.5.1997: Graz

Gemeinsame Tagung der Österreichischen Gesellschaft für Gynäkologie und Geburtshilfe und der Bayerischen Gesellschaft für Geburtshilfe und Frauenheilkunde

Kontaktadresse: Prof. Dr. R. Winter, Präsident der ÖGGG, Vorstand der geb.-gyn. Univ. Klinik Graz, Auenbruggerplatz, A-8036 Graz

Tel. (0316) 3852201 oder 2150

Veranstaltungen der Arbeitsgemeinschaften

A rbeitgemeinschaft für Uro-Gynäkologie

5.6.-6.6.1997: Wien

Urodynamik Seminar

(mit der AG Urogynäkologie der Deutschen Gesellschaft)

24.10.-25.10.1997: Innsbruck Operatives Tutorium

A rbeitsgemeinscha.fi fir Infektionen

1.5.-4.5.1997: Wien

III. European Congress for Infectious Diseases in Obstetric and

Gynecology

Arbeitsgemeinschafi für Endoskopie 8.5.-10.5.1997: Lockenhaus

11.9-13.9.1997: Villach

Pro und Kontra in der Gynäkologie

Arbeitsgemeinschafi für Kolposkopie

19.4.1997: Bozen

Gemeinsame Tagung mit Herrn Prim. Messini, KH Bozen

27.9.1997: KH Baden

(Organisation und Leitung durch Prim. Doz. Dr. F. Girardi,

Baden)

November 1997: AKH Wien (voraussichtlich eine weitere Veranstaltung)

15.5.-17.5.1997: Hotel Vienna Hilton, Wien

Menopause 97

Kontaktadresse: Austropa - Interconvention, Gabriele Kapper,

Postfach 30, A-1043 Wien, 
Tel. (0 222) 589 25-118, Fax (0 222) 5867127

21.5.-25.5.1997: Hotel Hilton, Wien

7th International Congress of Inborn Errors of

Metabolism/IEOM

Kontaktadresse: Mondial Congress, Faulmanngasse 4, A-1040 Wien, Tel. (0 222) 588 04-0, Fax (0 222) 5869185

24.5.1997: Steyr

Wiss. Sitzung der Österr. Gesellschaft für Prä- und

Perinatale Medizin

Kontaktadresse: Österr. Ges. f. Prä- u. Perinatale Medizin, II. Univ. Frauenklinik, Spita $1 / 8$ asse 23, A-1090 Wien

25.5.-29.5.1997: Salzburg

VI. International Congress of Andrology

Kontaktadresse: Prof. Dr. J. Frick, Abt. für Urologie, LKA Salzburg, Müllner Hauptstr. 48, A5020 Salzburg

11.6.-15.6.1997: Saalfelden

5. Symposium Medizin. Videof ilm

Kontaktadresse: EMB - Eva Maier Congress, Postfach 46, A-1095 Wien, Tel. (01) 4080059, Fax (01) 4022030

9.10.-12.10.1997: Hofburg Wien

IV. European Congress on Menopause

Kontaktadresse: Austro-Interconvention, G. Kapper, A-1042 Wien, Tel. (0 222) 58925 118, Fax (0 222) 5867127

18.10.1997: Zell am See

Wiss. Sitzung der Österr. Gesellschaft für Prä- und

Perinatale Medizin

Kontaktadresse: Österr. Ges. für Prä- u. Perinatale Medizin, II. Univ. Frauenklinik, Spita $1 / 8$ asse

23, A-1090 Wien

23.10.-25.10.1997: Wien

Österr. Ärztekongress, Van-Swieten-Tagung

Kontaktadresse: Wiener Medizinische Akademie, Alser Str. 4, A-1090 Wien, Tel. (0 222) 40513 83 12, Fax (0 222) 405138323

24.10.-28.10.1997: Salzburg

14. Jahrestagung der Österr. Gesellschaft für Senologie

Kontaktadresse: Prim. Dr. C. Menzel, LKA Salzburg, Müllner Hauptstr. 48, A-5020 Salzburg

6.12.1997: Horn

Wiss. Sitzung der Österr. Gesellschaft für Prä- und

Perinatale Medizin

Kontaktadresse: Österr. Ges. für Prä- und Perinatale Medizin, II. Univ. Frauenklinik, Spitalgasse 23, A-1090 Wien

Ausschreibungen

Hofrat-Erwin-Domanig-Stipendium

Anton-von-Eiselsberg-Preis

Der von der Van-Swieten-Gesellschaft gestiftete und gemeinsam mit der Österreichischen Ärztekammer zur Verleihung gelangende Anton-von-Eiselsberg-Preis beträgt für das laufende 
Jahr ATS 100.000.- und wird hiermit ausgeschrieben. Er ist in erster Linie für junge Kolleginnen und Kollegen gedacht, da der Preis der Förderung des wissenschaftlichen Nachwuchses dienen soil.

Österreichische Ärzte sind berechtigt, Arbeiten einzureichen. Die Arbeiten sollen die Ergebnisse eigener wissenschaftlicher, experi-menteller oder klinischer Untersuchungen aus einem Fachgebiet der Medizin zum Gegenstand haben.

Bei Arbeiten, die nur im Manuskript vorliegen, muss der Nach-weis erbracht werden, dass sie von einer Fachzeitschrift zur Publika-tion angenommen worden sind. Bereits publizierte Arbeiten können nur angenommen werden, wenn sie nach dem 30. April 1996 erschienen sind. Habilitationsschriften, sowie Arbeiten, die schon für eine andere Preisausschreibung eingereicht wurden, sind von der Einreichung ausgeschlossen.

Der eingereichten Arbeit (Original mit 5 Kopien) ist eine Erklä-rung des Autors beizuschliessen, dass alle an dem Zustandekommen der Arbeit beteiligten wissenschaftlichen Mitarbeiter (einschliesslich Biologen, Chemiker, Psychologen, Statistiker usw.) im Titel oder in Fussnoten oder sonst in irgendeiner Weise genannt sind. Klinik- und Institutsvorstände bzw. Primarärzte oder Leiter von Abteilungen (Departments) werden ersucht, selbst keine Arbeiten einzureichen. Wissenschaftlichen Arbeiten, welche nicht in deutscher, sondern in englischer Sprache erschienen sind, muss eine deutsche Zusammen-fassung beigefügt werden.

Die Einreichung hat bis spätestens 30. Juni 1997 (Datum des Poststempels) an den Sekretär der Van-Swieten-Gesellschaft, Univ.-Prof. Dr. E. Reinold, p.A. Wr. Med. Akademie, z.Hd. Fr. Silvia Kon-stantinou, Alserstrasse 4, A-1090 Wien, zu erfolgen.

Die Van-Swieten-Gesellschaft, Verein Österreichischer Ärzte, vergibt nach einem jährlich zu fassenden Vorstandsbeschluss, unter Berücksichtigung der jeweiligen finanziellen Gegebenheiten nach entsprechender Ausschreibung ein Stipendium, das nach dem Mitbegründer und Ehrenpräsidenten der Gesellschaft, Hofrat Erwin Do-manig, benannt ist.

Dieses Stipendium soil in erster Linie dem Kennenlernen und der praktischen Aneignung von neuen Methoden in der medizinischen Diagnostik und Therapie im Rahmen eines Auslandsaufenthaltes dienen; es darf nur an Nachwuchskräfte (Einzelpersonen) verliehen werden, die nach Werdegang und wissenschaftlicher Eignung die Gewähr dafür bieten, dass ein im Interesse des medizinischen Fort-schrittes in Österreich fruchtbares Ergebnis zu erwarten ist. Die Ausschreibung erfolgt nach der 1. Vorstandssitzung des jeweiligen Präsidentenjahres durch das Sekretariat.

Die Bewerber richten, entsprechend den Ausschreibungsbedin-gungen, ihr Ansuchen an das Sekretariat der Van-Swieten-Gesellschaft mit exakter Angabe des angestrebten Forschungsziels. Dem Ansuchen sind beizuschliessen:

Curriculum vitae.

Verzeichnis der wissenschaftlichen Publikationen.

Detaillierte Angaben über die mit dem Stipendium verbunde-ne Zielsetzung sowie über Ort, Zeit, Dauer und finanzielle Erforder-nisse.

Empfehlungen des Vorstandes der zuständigen Klinik (Abtei-lung, Institut).

Einreichungsberechtigt sind Nachwuchskräfte an österreichischen Kliniken, Abteilungen oder Instituten, welche im Besitz der österreichischen Staatsbürgerschaft und der Mitgliedschaft an der Van-Swieten-Gesellschaft sein müssen. Für die Vergabe des Stipen-diums setzt der Vorstand der Van-Swieten-Gesellschaft ein vier- bis sechsköpfiges Gremium unter dem Vorsitz des Präsidenten oder eines von ihm bestimmten Vertreters ein. Dieses Gremium entschei-det mit 
Stimmenmehrheit unter Ausschluss des Rechtsweges; bei Stimmengleichheit steht dem Vorsitzenden das Dirimierungsrecht zu.

Nach Abschluss der gefórderten Studien ist dem Vorstand der Van-Swieten-Gesellschaft ein schriftlicher Erfahrungsbericht zu er-statten. Anlässlich der Jahrestagung der Van-SwietenGesellschaft hat der Stipendiat über Einladung des Präsidiums ein entsprechen-des Kurzreferat zu halten.

Ende der Einreichungsfrist: 30. Juni 1997 (Datum des Poststempels)

Entsprechende Ansuchen sind an Herrn Univ.-Prof. Dr. E. Reinold, Sekretär der Van-SwietenGesellschaft, p.A. Wr. Med. Akademie, z.Hd. Fr. S. Konstantinou, Alserstrasse 4, A-1090 Wien, $\mathrm{zu}$ richten.

230

Mitteilungen aus Österreich 